\title{
Monitoring of postural sway with a head-mounted wearable device: effects of gender, participant state, and concussion
}

This article was published in the following Dove Press journal:

Medical Devices: Evidence and Research

\author{
Scott T Grafton' \\ Andreas B Ralston ${ }^{2}$ \\ John D Ralston ${ }^{2}$
}

'Department of Psychological \& Brain Sciences, UC Santa Barbara, Santa

Barbara, CA 93106-9660, USA; ${ }^{2}$ Clinical Studies Department, Protxx Inc, Menlo Park, CA, 94025-4317, USA
Correspondence: John D Ralston Protxx Inc, Menlo Park, CA 94025-43I7, USA

Tel +I 6502158418

Email john.ralston@protxx.com
Objective: To assess the utility of a head-mounted wearable inertial motion unit (IMU)based sensor and 3 proposed measures of postural sway to detect outliers in athletic populations at risk of balance impairments.

Methods: Descriptive statistics are used to define a normative reference range of postural sway (eyes open and eyes closed) in a cross-sectional sample of 347 college students using a wireless head-mounted IMU-based sensor. Three measures of postural sway were derived: linear sway power, eyes closed vs eyes open sway power ratio (Ec/Eo ratio), and weightbearing asymmetry (L-R ratio), and confidence intervals for these measures were calculated. Questionnaires were used to identify potentially confounding state variables. A prospective study of postural sway changes in 47 professional, college, and high school athletes was then carried out in on-field settings to provide estimates of session-to-session variability and the influence of routine physical activity on sway measures. Finally, pre-post-injury changes in sway are measured for a participant who was diagnosed with a concussion.

Results: Despite the heterogenous population and sampling environments, well-defined confidence intervals were established for all 3 sway measures. Men demonstrated significantly greater sway than women. Two state variables significantly increased sway: the use of nicotine and prescription medications. In the athletes, session-to-session variability and changes due to routine physical activity remained well within $95 \%$ confidence intervals defined by the cross-sectional sample for all 3 sway measures. The increase in sway power following a diagnosed concussion was more than an order of magnitude greater than the increases due to session-to-session variability, physical activity, or other participant state variables.

Conclusion: The proposed postural sway measures and head-mounted wearable sensor demonstrate analytic utility for on-field detection of abnormal sway that could be potentially useful when making remove-from-activity and return-to-activity decisions for athletes at risk of impact-induced balance impairments.

Keywords: wearable sensor, balance impairments, postural sway, concussion

\section{Introduction}

Measures of postural sway are important for assessing abnormalities of the vestibular system resulting from a wide range of injury, medical-treatment, and agerelated balance impairments, including concussions, ${ }^{1-4}$ subconcussive events, ${ }^{5-8}$ industrial accidents, ${ }^{9}$ combat blast, ${ }^{10,11}$ anesthesia, ${ }^{12}$ Parkinson's disease, ${ }^{13,14}$ Alzheimer's disease, ${ }^{15}$ and multiple sclerosis. ${ }^{16}$ The particular vulnerability of balance is not surprising given the structural complexity of the labyrinthine 
vestibular apparatus, the risk of damage to otolith or semicircular canal hair cells due to high acceleration forces and other factors, and the overall complexity of multisensory integration that combines visual, proprioceptive, vestibular, and efferent motor inputs and outputs to maintain body position. ${ }^{10,17-19}$ The vestibular system is also essential for gaze stabilization and visuomotor coordination, and persistent vestibular impairment due to head impacts has been observed to cause deficits of athletic performance and increase the risks of falls and future sports-related orthopedic injuries. ${ }^{20-25}$

While severe impairments of balance can be identified by qualitative variations of Romberg testing and the BASS and BEST balance tests, instrumented measurements provide enhanced sensitivity for detecting less severe vestibular abnormalities. ${ }^{26}$ Tools such as force plates, routinely used for balance assessment in clinical environments, are impractical to implement on-field in most athletic settings and are inefficient for assessing an entire team in parallel during games or practices where time is limited. Force plate sway metrics are all derived from body center-ofmass (COM) trajectory parameters calculated from foot center-of-pressure (COP) data, modeling the body as an inverted or articulated pendulum and requiring multiple biophysical assumptions. $^{27-29}$ There is still no consensus in the literature as to the accuracy and physiological interpretation of these many sway metrics. ${ }^{30}$ As an alternative, inertial measurement unit (IMU)-based sensors worn on the body have been shown to provide valid and sensitive metrics of postural sway ${ }^{31,32}$ that correlate well with force plates. $^{33,34}$ Here, we focus on a head-mounted wireless sensor that can be rapidly deployed in the on-field setting. Head-mounted IMUs are increasingly used to continuously monitor intensity and frequency of head impacts during athletic events. ${ }^{35}$ In addition, head-mounted IMU devices can be used to evaluate postural sway, ${ }^{36}$ a proxy for vestibular integrity, analogous to what has been accomplished with body-mounted IMUs (which do not track head impacts) and force plates.

In the current paper, we establish the utility of a simple and pragmatic head-mounted IMU measure of sway that does not require biophysical models or complex computation. Our goal was to test if this device could be used to obtain standardized, practical, on-field measures of postural sway in less than one minute, with the acquisition supervised by non-expert operators. Standardization under these variable conditions could help to more effectively quantify potential vestibular impairment in a variety of populations and measurement settings. We characterize the performance of this wearable head-mounted IMU-based sensor for measuring postural sway in four independent populations. First, we tested a large sample of otherwise healthy college undergraduates to establish normative values of sway across a general population of young adults. To increase the generalizability of the findings to non-laboratory settings, the measures were obtained across heterogeneous campus environments and they were obtained by undergraduate research assistants rather than expert laboratory technicians. Participants in this phase of the study also completed a questionnaire to identify state variables that might influence balance as measured with the device. We hypothesized that a variety of lifestyle-related variables such as prior exercise, sleep, food consumption, use of medications, recreational drugs, nicotine, and alcohol might influence balance performance. To expedite data acquisition, independent morphometric properties such as height and weight, which have been previously shown to influence measurements of sway, were not replicated in this study. Descriptive statistics from the measures of the large sample were then used to establish normative confidence intervals. These could then be used for defining outliers that would suggest a participant had vestibular dysfunction, applicable to a general population, irrespective of morphometrics.

To further evaluate the robustness of the benchmark measures defined from the cross-sectional sample, we tested session-to-session variability of the above sway measures for a men's professional soccer team. To our knowledge, evaluations of the within-session and between-sessions reliability of sway parameters measured by inertial sensors have only been reported for small experimental populations, ${ }^{32,34}$ and none of these studies utilized head-mounted sensors. We also evaluated the influence of routine physical activity ${ }^{37}$ during typical training sessions on measures of postural sway for college women's soccer and volleyball teams. Finally, to further demonstrate the potential utility of a head-mounted IMU for on-field quantitative assessment of balance impairment, we present a case study of 6 varsity high-school football players for whom data were acquired before and after a practice during which one of the athletes sustained a concussion.

\section{Methods}

\section{Participants}

Sample 1 (Normative measures in a general population): The cross-sectional study of college undergraduates was performed on campus at the University of California at Santa 
Barbara (UCSB). A total of 347 participants were measured by 10 undergraduate research assistants and included in the study (196 women, 150 men, one undeclared, mean age 21.1 \pm 0.32 ). Inclusion criteria was anyone aged 18 and older. Exclusion criteria were no major medical disease or ongoing illness (by self report). The RAs were also advised to not recruit participants who might be intoxicated based on behavior or self report. However, no formal testing of blood alcohol levels was obtained to screen subjects.

Sample 2 (Session-to-session variability of postural sway): This was a targeted recruitment of 22 healthy male athletes (aged 22.6 \pm 3.6 ) from a professional soccer team, participating in up to 15 measurement sessions before practices. All data were acquired at the team's practice facility over a 37-day period.

Sample 3 (The influence of physical activity on postural sway): This was a targeted recruitment of women from two college athletic teams. 11 women's soccer players (aged 19.9 \pm 0.3 ) were tested before and after practice during 13 separate sessions over a 40-day period, and 8 women's volleyball players (aged 19.9 \pm 0.4 ) were tested before and after practice during 15 separate sessions over a 43-day period. All data were acquired at their respective practice facilities.

Sample 4 (Repeated measures in a setting with high risks for concussion): This was a targeted recruitment of 6 members of a varsity high school football team, all 18 years of age, during regularly scheduled practices. All data were obtained on field or in the team meeting room.

\section{Ethical considerations}

Sample 1 participants provided informed consent in accordance with the UCSB IRB and were paid $\$ 2$ for their participation. Professional, college and high school athlete testing was carried out under Western IRB Study Number 1188786, and all participants provided written informed consent.

\section{Measurements}

Sample 1 participants in the cross-sectional study were tested with a wearable IMU sensor housed in a removable plastic shell and positioned superior to the right ear by means of an athletic headband (Figure 1A). Postural sway measurements were carried out in flat-soled shoes or socks, and on a hard surface. Sample 2-4 athletes were tested with a miniaturized wearable IMU sensor mounted on the skin superior to the right ear by means of a disposable 2-sided medical adhesive patch (Figure 1B). Both sensors incorporate the same processor, memory, Bluetooth communications, and IMU module, capable of measuring postural sway, single head impacts, and cumulative head impact loads.

Participants were instructed to stand erect with feet pressed together, and after a 5-second count down, data were obtained for $30 \mathrm{~s}$ with eyes open and after a brief pause a second set of data were obtained for $30 \mathrm{~s}$ with eyes closed. Filtering of the raw accelerometer data was carried out directly on the IMU. Measures of linear motion along 3 axes were sampled at $100 \mathrm{~Hz}$ and transmitted via Bluetooth to the investigator's iPhone or iPad and uploaded to a cloud database for subsequent analyses. The mobile app and cloud data platform support connectivity to an entire team of sensor-equipped athletes in parallel, providing a significant streamlining of the athlete testing workflow. Cumulative sway motion at the head was quantified by calculating linear sway power directly from

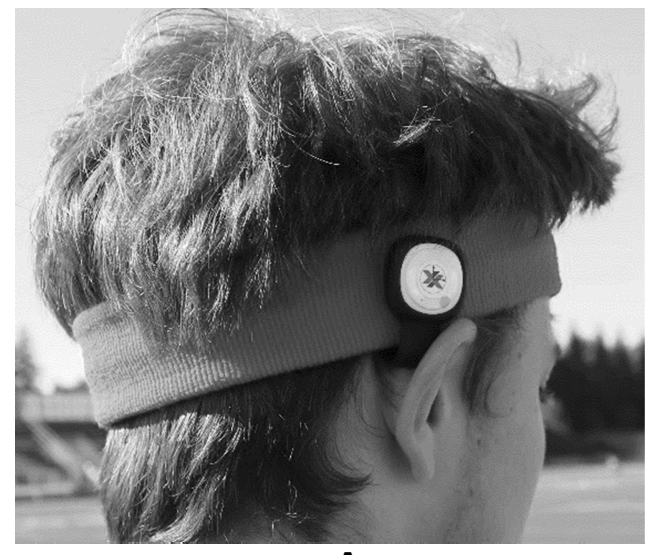

A

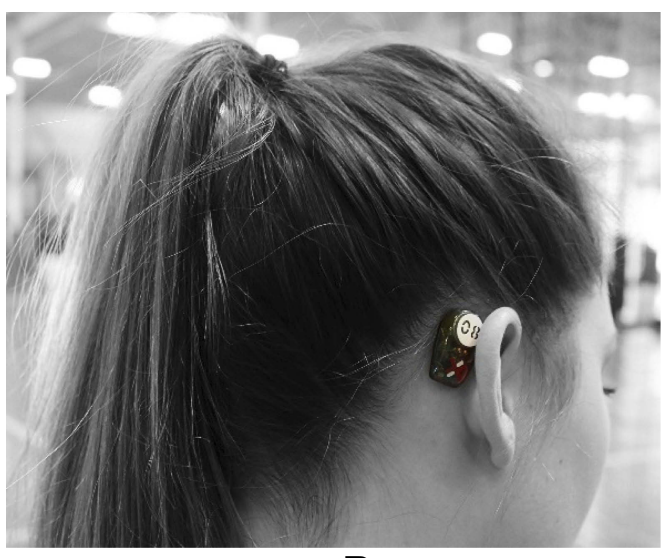

B

Figure I Combined head-impact and postural sway monitoring inertial motion unit sensors (A) worn with an athletic headband; and (B) attached to the skin using an adhesive patch. 
the filtered accelerometer data and assuming a brain of mass $1.4 \mathrm{~kg}$ moving under these accelerations. The primary measures of postural stability were:

1. overall sway power with eyes open and closed (in Watts)

2. the proportional change of sway power (eyes closed/ eyes open, referred to in herein as Ec/Eo ratio)

3 . the bias of sway power in the left-right direction (relative offset of lateral sway, referred to herein as L-R ratio) with eyes open and separately for eyes closed

4. the bias of sway power in the front-back direction (relative offset of forward-backward sway, referred to herein as F-B ratio) with eyes open and separately for eyes closed.

In addition to the above accelerometer measures, each participant in Sample 1 (the cross-sectional study) completed an online questionnaire that queried their history of prior concussions and a set of state variables related to sleep, exercise, diet, nicotine, medications, recreational drugs, alcohol, and caffeine.

\section{Data analysis}

All analyses were performed using the statistical software $\mathrm{R}$, version 3.3.2. As a first step, distributions of sway measures from Sample 1 were first tested for normality by the Shapiro-Wilk statistic with the R-function shapiro.test. The power measurements deviated significantly from a normal distribution (as shown in Figure 2).

\section{Descriptive statistics}

Because of the non-normal distributions, confidence intervals were derived using a bootstrap method with the $\mathrm{R}$ function boot.mean.
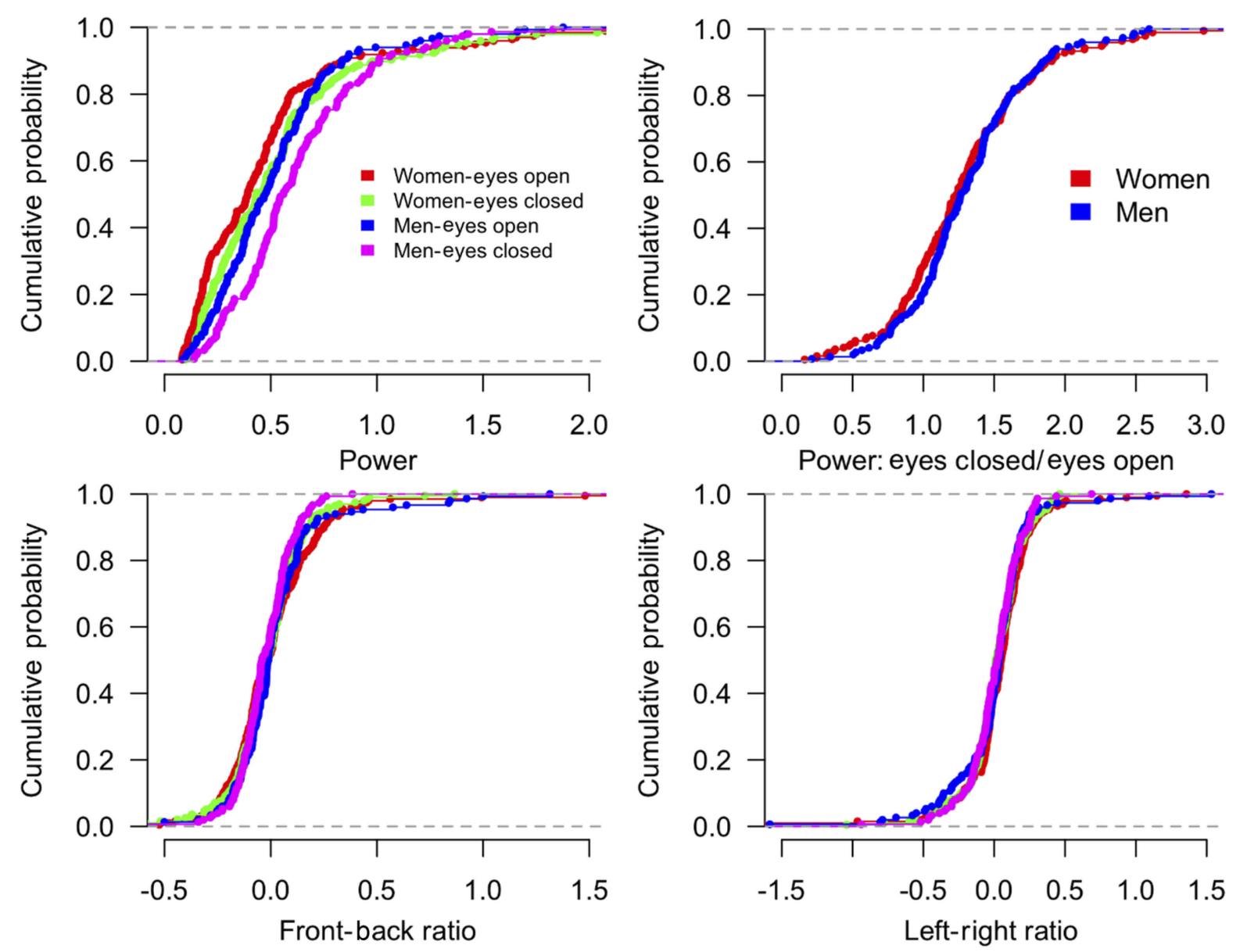

Figure 2 Cumulative distributions for different measures of postural stability, plotted as a function of eye state and gender. Measures of sway power (in Watts) were significantly different for eyes open (Eo) versus eyes closed (Ec) and as a function of gender. After normalization, the ratio of sway power with Ec/Eo did not differ as a function of gender. The Front-back sway power ratio was significantly different: with eyes closed participants moved more posteriorly. There were no differences in the left-right sway power ratio as a function of gender or eye state. 


\section{Analysis of variance testing}

To test for effects of gender and the state variables obtained by questionnaire, the sway power data were first $\log$ transformed. Null hypothesis testing was performed by $t$-test ( $\mathrm{R}$ function t.test), ANOVA ( $\mathrm{R}$ function anova), and for unbalanced designs involving repeated measures ANOVA with linear mixed effects models ( $\mathrm{R}$ functions aov and lme). To test for an increased bias in the lateral axis independent of left-right directions, the absolute value of the L-R ratio was used in the ANOVA to test for lateral bias. Because the goal of this survey experiment was to identify any potential factors that might impact measures of sway, we report uncorrected $p$-values (0.05 threshold). All error bars reflect standard error of the mean.

\section{Results}

\section{Sample I - Cross sectional sample of a college population}

The primary objective with this sample was to develop a normative description of sway measures, organized by significant state and trait variables.

\section{Gender and visual feedback}

As shown in Figure 2 and summarized in Table 1, both men and women demonstrated significantly greater sway with eyes closed than open, consistent with the classic studies of Romberg and subsequent balance researchers: ${ }^{38,39} \mathrm{~F}(1,344)=85.03, p<0.0001$. In addition, clear differences of sway as a function of gender were observed: $\mathrm{F}(1,344)=10.95, p<0.001$. There was no interaction between the state of the eyes and gender on the sway power measures $\mathrm{F}(1,344)=1.42, p>0.23$. There was a significant bias in the F-B ratio when the eyes were closed compared to when they were open $\mathrm{F}(1,344)=5.90$, $p<0.02$; participants tend to move more posteriorly with eyes shut. However, there was no difference of the F-B ratio as a function of gender or the interaction of eye state and gender. There were no differences of L-R ratios as a function of eye state, gender, or their interaction. We also normalized the measured sway power values by calculating the Ec/Eo ratio, a value that is normally greater than 1 . There were no gender differences for this ratio $\mathrm{t}(340)=0.54, p>0.59$. Given these aggregate results, we stratified the different measures of sway as a function of gender and eye state, then estimated the $95 \%$ confidence intervals for each measure. As shown in Table 1, the $95 \%$ confidence intervals for the sample means are remarkably tight for all measures, suggesting that it is feasible to identify individuals as outliers after accounting for eye state and gender. All subsequent statistical analyses were performed separately for eyes open and closed and with gender as a factor.

\section{History of concussion}

A total of 32 participants in Sample 1 reported having had experienced a previous concussion. While two had occurred within 3 months, the remainder had occurred at least 12 months prior to this study, with many extending back more than 5 years prior to this study encounter. Thus, the pooled results reflect the influence of remote concussion on sway. There were no significant differences observed for any of the sway measures. However, these results are based on self-reporting from a small pool of participants, so the interpretation of potential changes in postural sway after head injury merit further investigation.

\section{Participant state}

A wide variety of environmental or state variables can potentially influence postural stability. As shown in Table 2 and Figure 3, of the entire set of state variables

Table I Measurements of sway power and sway power asymmetry (front/back ratio and left/right ratio) as a function of participant gender

\begin{tabular}{|c|c|c|c|c|c|c|c|c|c|c|}
\hline \multirow[t]{2}{*}{ Eyes } & \multirow[t]{2}{*}{ Gender } & \multicolumn{2}{|l|}{ Power } & \multirow[b]{2}{*}{$\boldsymbol{p}$} & \multicolumn{2}{|c|}{ Front/back ratio } & \multirow[b]{2}{*}{$p$} & \multicolumn{2}{|c|}{ Left/right ratio } & \multirow[b]{2}{*}{$p$} \\
\hline & & Mean & $95 \% \mathrm{Cl}$ & & Mean & $95 \% \mathrm{Cl}$ & & Mean & $95 \% \mathrm{Cl}$ & \\
\hline \multirow[t]{2}{*}{ Open } & Men & 0.519 & $0.468-0.569$ & $*, \Delta$ & 0.023 & $-0.016--0.062$ & $x$ & 0.005 & $-0.045-0.055$ & ns \\
\hline & Women & 0.487 & $0.419-0.556$ & & 0.014 & $-0.022-0.050$ & & 0.029 & $-0.020-0.078$ & \\
\hline \multirow[t]{2}{*}{ Closed } & Men & 0.628 & $0.573-0.683$ & & -0.028 & $-0.05 \mathrm{I}--0.005$ & & 0.007 & $-0.025-0.039$ & \\
\hline & Women & 0.551 & $0.483-0.619$ & & -0.013 & $-0.039-0.014$ & & 0.010 & $-0.020-0.041$ & \\
\hline \multirow[t]{2}{*}{ Closed/open } & Men & 1.313 & $1.244-1.383$ & ns & & & ns & & & ns \\
\hline & Women & 1.286 & 1.214 & & 1.358 & & & & & \\
\hline
\end{tabular}

Notes: $*_{p}<0.0001$ main effect of eyes. $\Delta p<0.001$ main effect of gender. $x p<0.002$ main effect of eyes. Separate measures were obtained with the eyes open and eyes closed. Abbreviation: ns, not significant. 


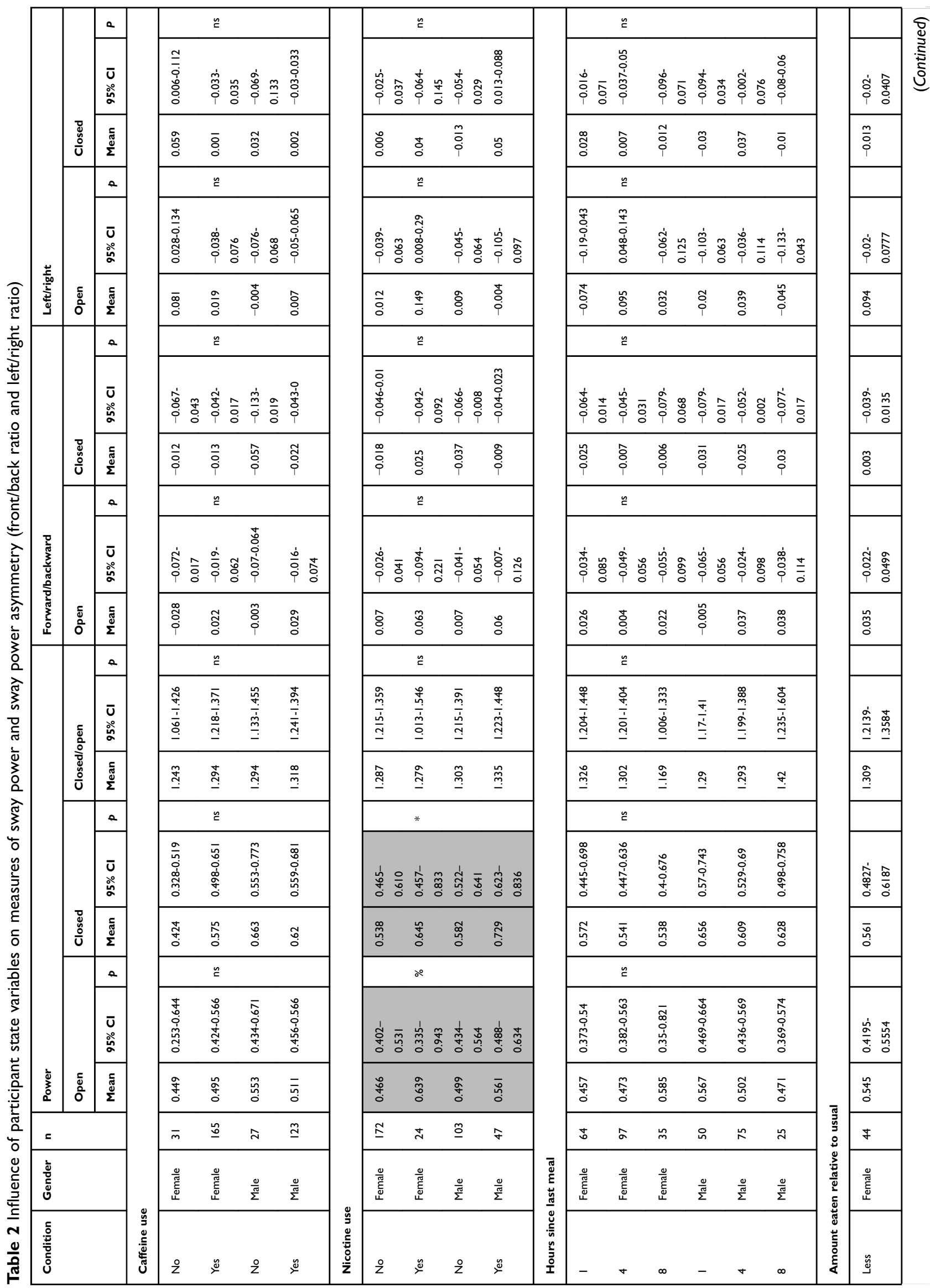




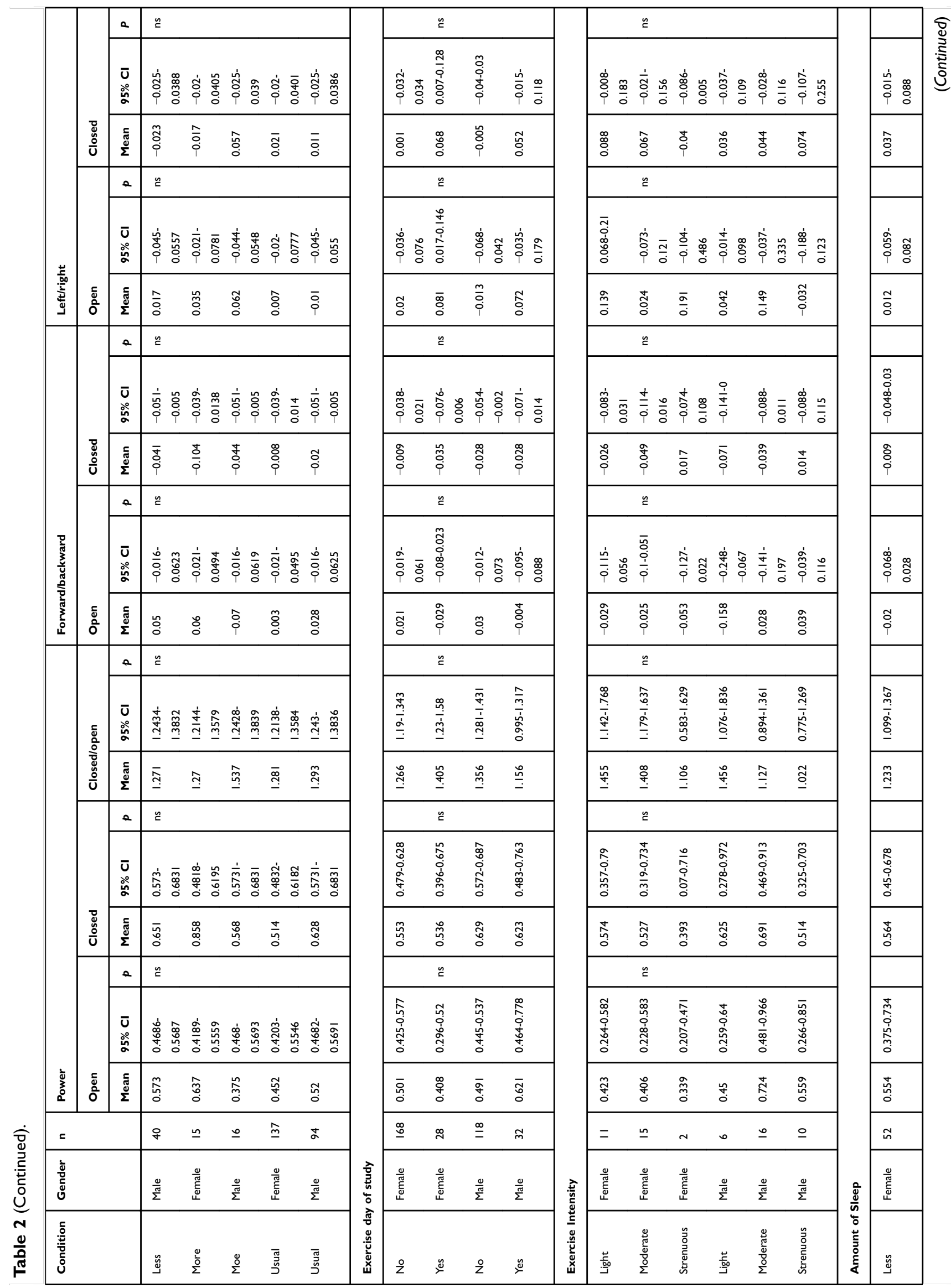




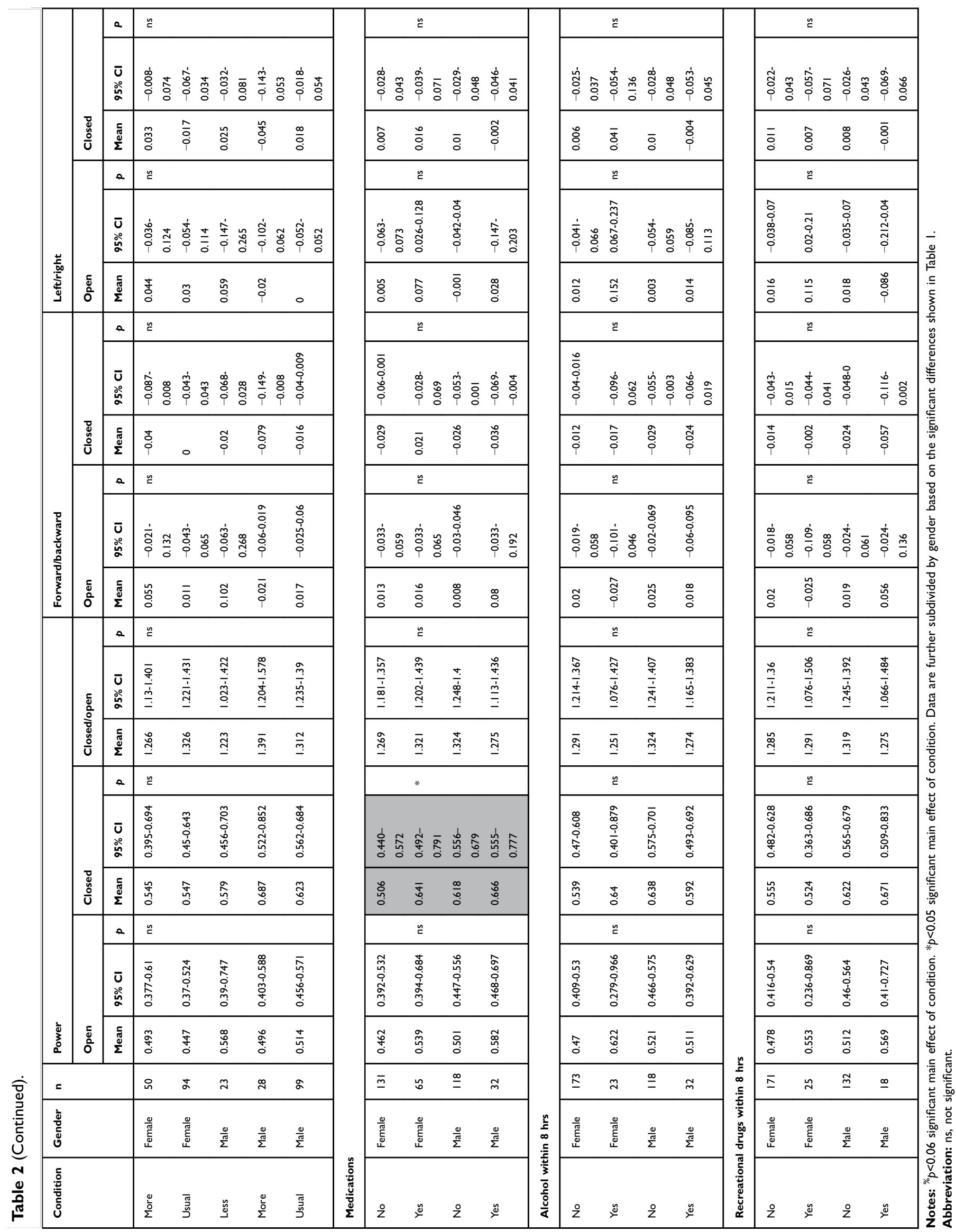




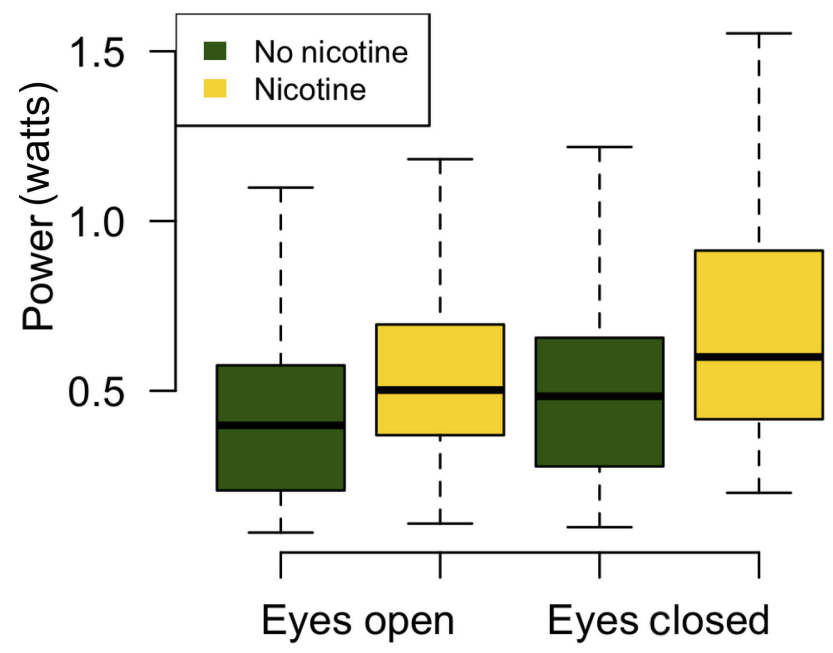

Figure 3 The influence of nicotine use (within I-8 hrs) on postural sway.

that we queried, the most important single factor influencing the power estimates of postural sway was the use of nicotine. Consumption of any nicotine products significantly increases postural sway in both men and women and whether the eyes were open $(\mathrm{F}(1,342)=3.49, p<0.06)$ or closed $(\mathrm{F}(1,342)=5.249, p<0.03)$. There was no interaction of the nicotine effect with gender. Nicotine did not cause a significant difference in the three ratio measures (Ec/Eo, L-R, F-B). On further analysis of only the nicotine users, we found that time since last nicotine $(1,2,4,8 \mathrm{hrs})$ did not influence the increase in power, suggesting that nicotine causes a long-lasting decrement of postural stability. The other state variable that influenced postural sway was use of prescription medications in the previous $48 \mathrm{hrs}$. While the effect was not significant with eyes open, sway was significantly increased with eyes closed $(\mathrm{F}(1,342)$ $=4.16, p<0.05)$. Because this was not a clinical study with HIPAA clearance, we do not know the specific prescription medications that were taken. Of the remaining queried state variables, caffeine, diet, exercise sleep, alcohol consumption, and recreational drug use had no observed effect on sway measures.

\section{Sample 2 - Repeated measures from a men's professional soccer team}

The primary objective with this sample was to evaluate session-to-session variability of the postural sway measurements. To assess this, 22 professional men's soccer players were tested with eyes open and eyes closed before
A

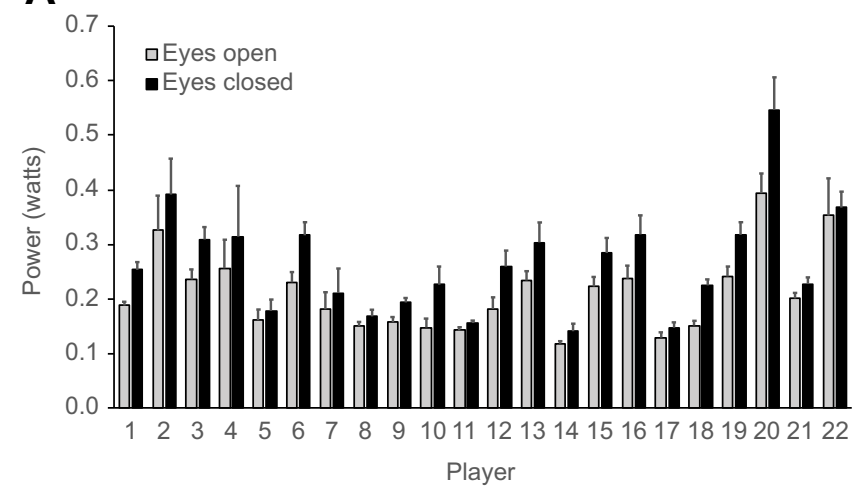

B

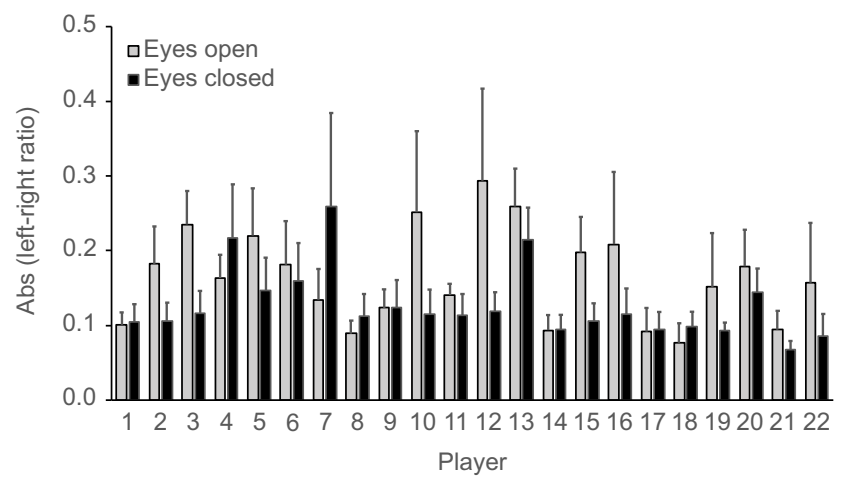

C

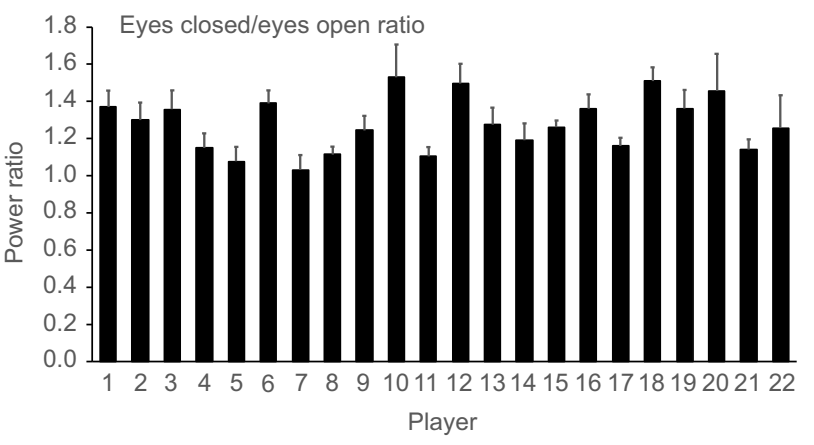

Figure 4 Repeated measures of sway in 22 men's professional soccer players recorded over 15 sessions spanning 37 days. (A) Sway power; (B) Bias in lateral sway; (C) Eyes closed/eyes open sway power ratio.

Abbreviation: L-R, left-right. 
practice in 15 separate sessions over a 37-day period. As shown in Figure 4, the average values of sway power (Figure 4A), L-R ratio (Figure 4B), and Ec/Eo ratio (Figure $4 C$ ) were entirely consistent with the previous large cross-sectional study and all values were well inside of $95 \%$ confidence intervals. (F-B ratio was not estimated in this sample). Similarly, as shown in Figure 5, the session-to-session variance was also low for sway power (Figure 5A), L-R ratio (Figure 5B), and Ec/Eo ratio (Figure $5 \mathrm{C}$ ), further supporting the reliability of the sway measures with this wearable device. The team's average coefficient of variation of postural sway power across all sessions was $32 \%( \pm 3.69)$ with eyes open and $35 \%( \pm 4.10)$ with eyes closed, and the ratio of Ec/Eo varied by $24 \%$ $( \pm 1.81)$. There was much greater variability in the L-R ratio, reaching $100 \%( \pm 7.83)$ with eyes open and $88 \%( \pm 5.6)$ with eyes closed. Examination of individual values reveals that this high variation is largely an effect of small numeric ratio values. In no case was a sway power or ratio value outside the $95 \%$ confidence interval defined by the larger independent cross-sectional sample.

\section{Sample 3 - Effects of physical activity in college women's soccer and volleyball}

The primary objective with this sample was to evaluate the impact of physical activity on postural sway measurements. Sway data was collected both before and after training sessions for a women's collegiate soccer team and a women's collegiate volleyball team. In these populations, we focused on overall sway power and L-R ratio as indicators of recent physical activity. As shown in Figure 6, the range of values is consistent with the other cohorts in this paper. For both sports, there is a significant effect of vision on sway power: it increases with eyes closed $\mathrm{F}(1,282)=26.6, p<0.0001$ for soccer, $\mathrm{F}(1,238)=26.9, p<0.0001$ for volleyball. Sway power also increased significantly after physical activity: $\mathrm{F}(1,282)$ $=13.8, p<0.0002$ for soccer, $\mathrm{F}(1,238)=11.8, p<0.0007$ for volleyball, but all values remained within the $95 \%$ confidence intervals defined by the large cross-sectional sample. There was no significant interaction between physical activity and vision. In contrast to sway power, neither team demonstrated a significant difference of L-R ratio after physical activity.
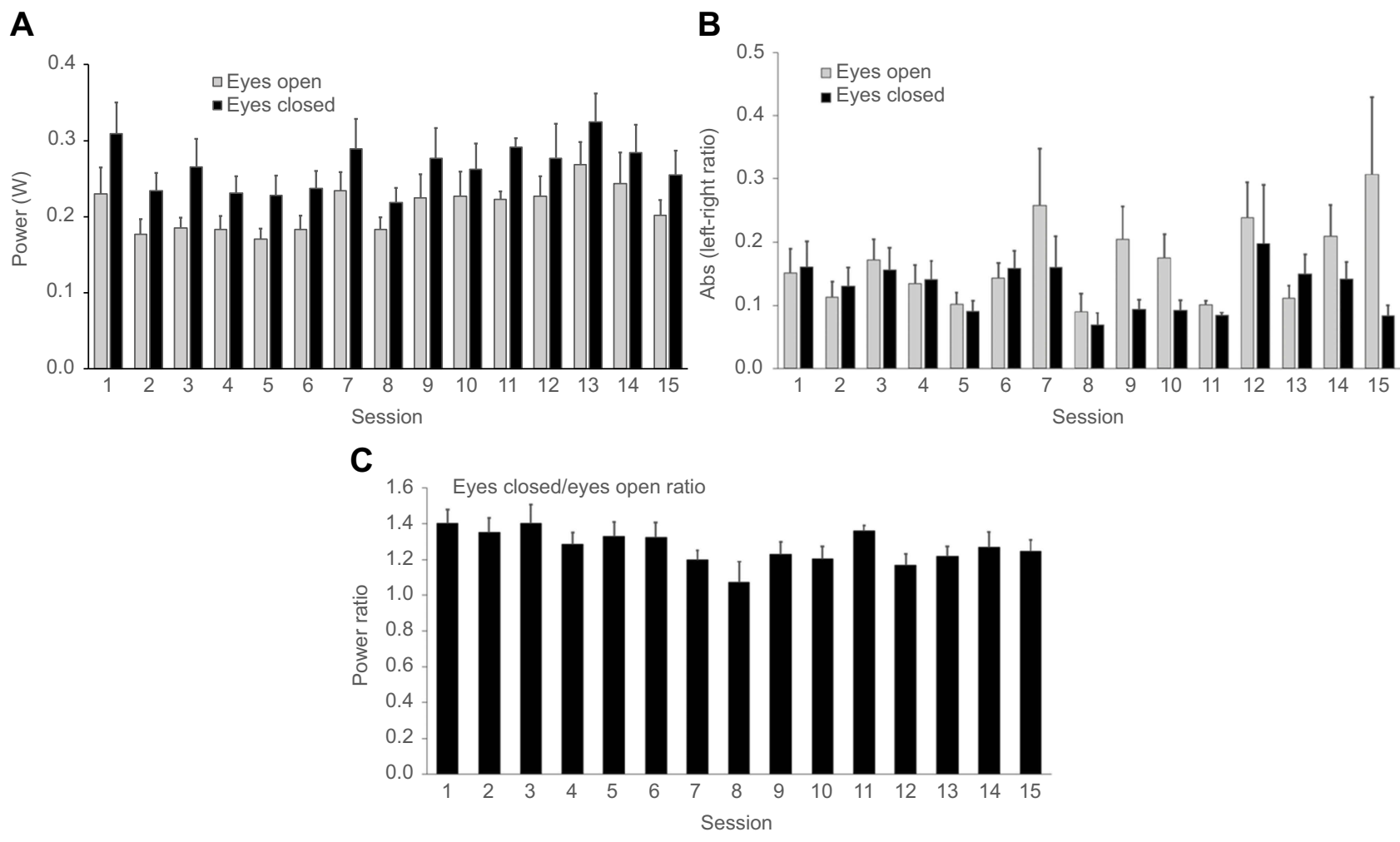

Figure 5 Variability of sway measurements from 22 men's professional soccer players recorded over 15 sessions spanning 37 days. (A) Sway power; (B) Bias in lateral sway; (C) Eyes closed/eyes open sway power ratio.

Abbreviation: L-R, left-right. 

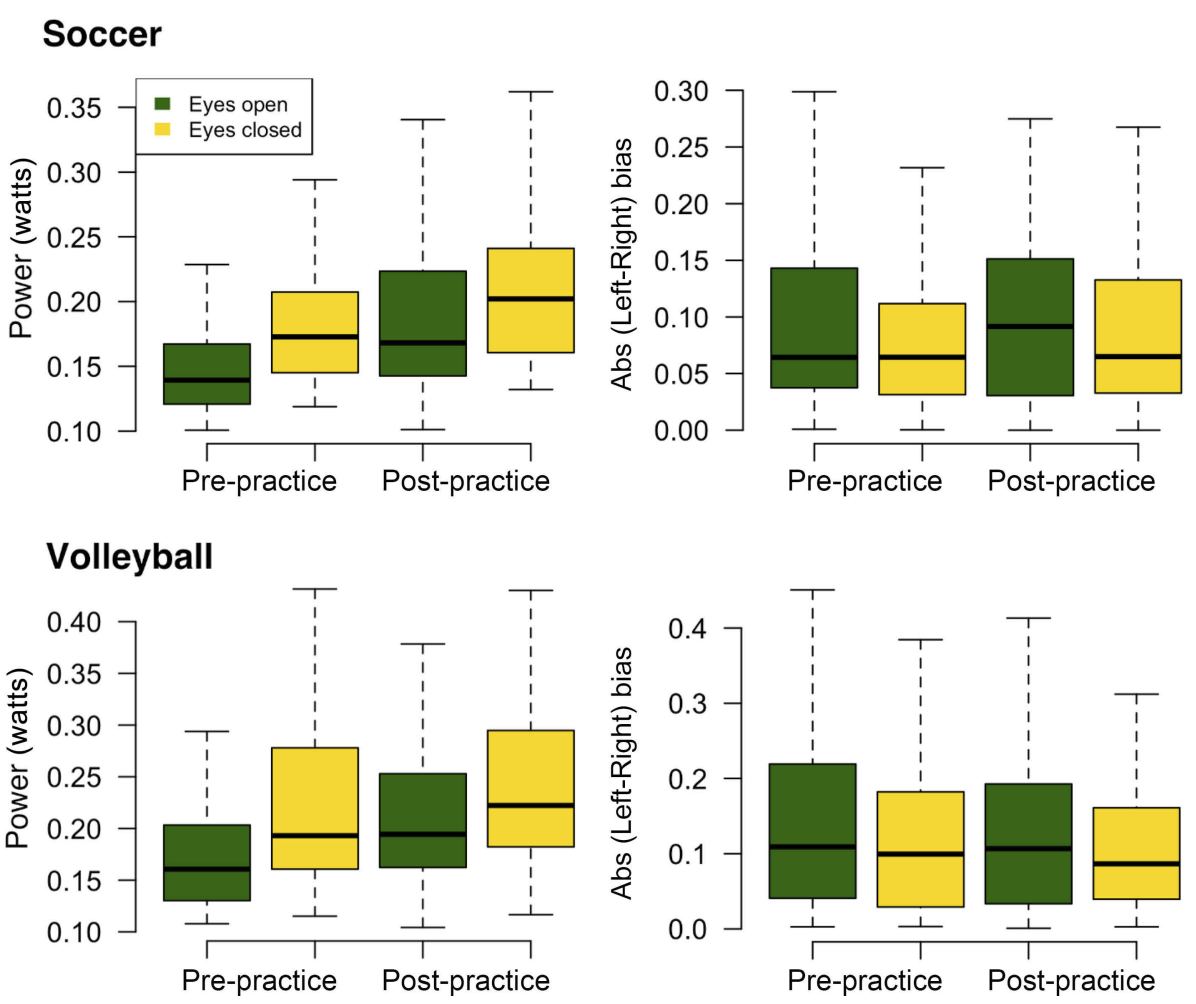

Figure 6 The effect of physical activity on sway in two sports. The measures were obtained in college women's soccer and volleyball teams and reflect repeated measures before and after training sessions over 6 weeks during the fall 2018 season. Sway power increases significantly after physical activity.

Abbreviation: L-R, Left-right

\section{Sample 4 - The effect of acute concussion on postural sway, a case report}

While testing the reliability and utility of the device in high school football, we had the opportunity to capture serial sway measures before and after an isolated concussion in a group of high school football players. We acquired serial pre- and postpractice postural sway measurements for six 18-year-old high school varsity football players who were being tracked as a group (all starters with significant impact exposure on a regular basis). Mid-season, one of the players was suspected during practice of having experienced a concussion, and subsequently diagnosed with a concussion by the team athletic trainer. As shown in Figure 7A, the concussed athlete's postpractice Eo and Ec sway powers both showed a 9-10-fold
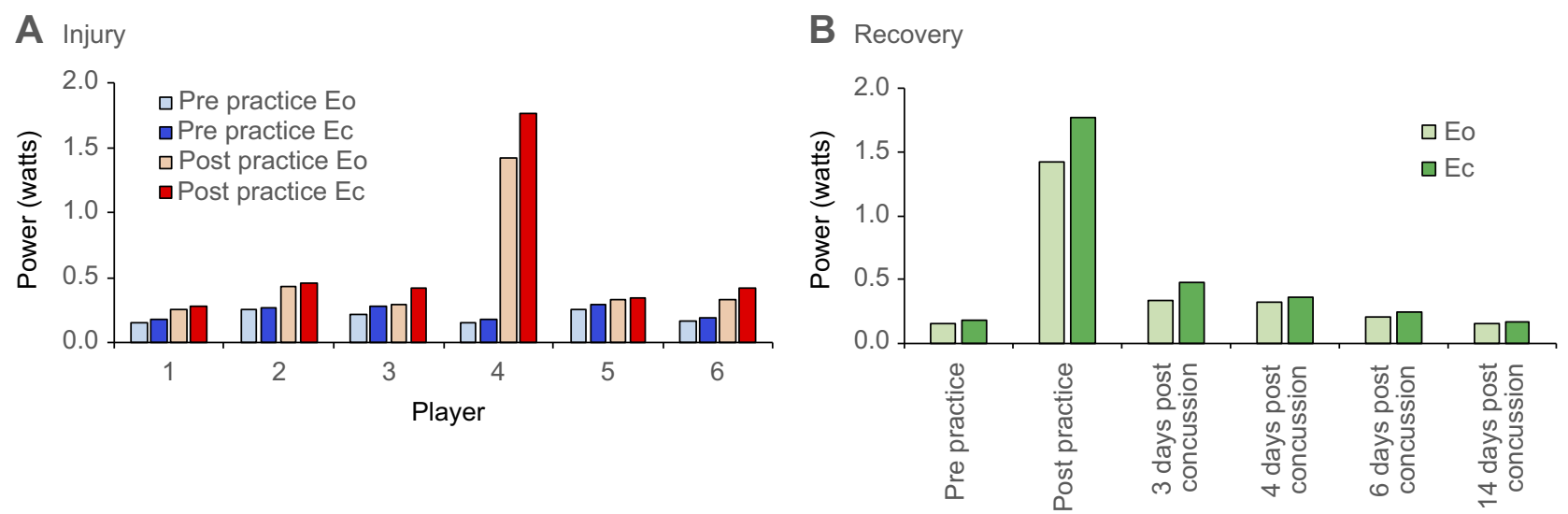

Figure 7 The effect of an acute concussion on sway power in a varsity high school football player. Player 4 sustained an in-practice concussion, leading to a significant increase of postural sway post practice (A). Over the next two weeks, there is a clear pattern of recovery to baseline (B). Abbreviations: Eo, eyes open; Ec, eyes closed. 
increase, more than an order of magnitude greater than the activity-related increases observed for the other five players, and more than an order of magnitude greater than the sessionto-session variability or the increases due to physical activity or other participant state variables observed in the other cohorts in this paper. The concussed athlete was subsequently managed according to the California Interscholastic Federation (CIF) concussion guidelines and return to play protocol. In parallel, repeated measurements of sway over the ensuing two weeks demonstrated the eventual recovery to baseline, as shown in Figure 7B.

\section{Discussion}

In this study, we demonstrate a head-mounted wearable IMU-based sensor, together with a set of postural sway measures that are sufficiently consistent, reproducible and sensitive for actionable assessments in both clinical and onfield environments. The device itself is small and unobtrusive enough to be worn by athletes of any age, any skill level, and in any sport. The $95 \%$ confidence intervals for normative values of the sway power, Ec/Eo ratio, and L-R ratio are remarkably tight for all measures, suggesting that it is feasible to identify individuals as outliers after accounting for eye state and gender. We also took into account many of the concomitant factors that potentially influence a healthy person's vestibular function. Nicotine was found to increase sway in both men and women, consistent with previous reports that nicotine can induce transient balance impairments. ${ }^{40}$ Analogous increases of sway were observed in the small subset of subjects taking prescription medicines. It should be noted however that while these state variables led to increased sway, the effect size was small and neither of the observed changes in our IMU-based measures of postural sway due to participant state exceeded the $95 \%$ confidence intervals. Similarly, session-to-session variability and the effects of routine physical activity did not lead to sessions with an abnormal increase of sway. These results establish the potential utility of this approach for detecting abnormal sway and quantifying natural variations that should be considered when making remove-from-activity and return-toactivity decisions, or when attempting to quantify the effects of sub-concussive head impacts. Indeed, the potential of the device for on-field injury detection and subsequent tracking of clinical recovery was clearly demonstrated in a case report of a high school athlete suffering a concussion. While limited to a single case, the results underscore a key point, that simple practical on-field measures of sway hold enormous potential for characterizing injury-related deficits of balance.
Such a solution is also applicable to a wide range of other populations at risk of balance impairments, including elderly people $^{41}$ industrial workers, ${ }^{9}$ and military personnel, ${ }^{10,11}$ and can be used by clinical and allied health care providers to: measure changes in postural sway for individuals or groups; identify individuals falling outside of normative ranges so that they can be proactively flagged for evaluation and any required further assessment and treatment; and track progress during recovery and rehabilitation.

\section{Conclusion}

This paper establishes the utility of a very simple and practical head-mounted IMU-based measure of postural sway that does not require the biophysical models or complex computations associated with force plates. The results demonstrate that the device can be used to effectively quantify potential vestibular impairment in a variety of populations and measurement settings, with the data acquisition supervised by non-expert operators. Since the device is headmounted, it can also monitor cumulative head impact exposures that cause balance impairments such as those observed via the postural sway measures. These dual-function measurements and related correlations between impact loads and balance impairments will be the subject of future studies.

\section{Acknowledgments}

The authors wish to thank Andreas Hauenstein for support in developing and maintaining the mobile app and cloud data platform used in this study, Jeremy Morkoch for carrying out postural sway measurements in Sacramento, and the Research Assistants who carried out the postural sway measurements at UCSB: Andrea Asatourian, Allison Chang, Clarissa Gooze, Sylvia Liu, Valeria Sevilla Mercado, Quynh Phan, Christian Quebral, Brady Rochon, and Christian Yang. We also thank the players, coaches, and training staff at the Sacramento Republic FC soccer team, William Jessup College women's soccer and volleyball teams in Rocklin, CA, and Menlo-Atherton high school varsity football team in Menlo Park, CA who participated in and supported this study. Protxx, Inc. provided the sensors, mobile app, and cloud data platform used in this study, and supported participant recruitment.

\section{Disclosure}

John Ralston is the founder and CEO of Protxx, Inc., and has a financial interest in the company. The authors report no other conflicts of interest in this work. 


\section{References}

1. Gurley JM, Hujsak BD, Kelly JL. Vestibular rehabilitation following mild traumatic brain injury. NeuroRehabilitation. 2013;32:519-528. doi:10.3233/NRE-130874

2. Chorney SR, Suryadevara AC, Nicholas BD. Audiovestibular symptoms as predictors of prolonged sports-related concussion among NCAA athletes. Laryngoscope. 2017;127:2850-2853. doi:10.1002/ lary. 26564

3. Guskiewicz KM, Ross SE, Marshall SW. Postural stability and neuropsychological deficits after concussion in collegiate athletes. $J$ Athl Train. 2001;36:263-273.

4. Fino PC, Nussbaum MA, Brolinson PG. Decreased high-frequency center-of-pressure complexity in recently concussed asymptomatic athletes. Gait Posture. 2016;50:69-74. doi:10.1016/j. gaitpost.2016.08.026

5. Caccese JB, Buckley TA, Tierney RT, Rose WC, Glutting JJ, Kaminski TW. Postural control deficits after repetitive soccer heading. Clin J Sport Med. 2018;1. doi:10.1097/ JSM.0000000000000709

6. Heick JD, Bay C, Dompier TP, Valovich McLeod TC. Relationships among common vision and vestibular tests in healthy recreational athletes. Int J Sports Phys Ther. 2017;12:581-591.

7. Hwang S, Ma L, Kawata K, Tierney R, Jeka JJ. Vestibular dysfunction after subconcussive head impact. $J$ Neurotrauma. 2017;34:8-15. doi:10.1089/neu.2015.4238

8. Miyashita TL, Diakogeorgiou E, Marrie K. The role of subconcussive impacts on sway velocities in division I men's lacrosse players. Sports Biomech. 2018;36:1-9. doi:10.1080/14763141.2018.1458892

9. Kincl LD, Bhattacharya A, Succop PA, Clark CS. Postural sway measurements: a potential safety monitoring technique for workers wearing personal protective equipment. Appl Occup Environ Hyg. 2002;17:256-266. doi:10.1080/10473220252826565

10. Akin FW, Murnane OD, Hall CD, Riska KM. Vestibular consequences of mild traumatic brain injury and blast exposure: a review. Brain Inj. 2017;31:1188-1194. doi:10.1080/02699052.2017.1288928

11. Pan T, Liao K, Roenigk K, Daly JJ, Walker MF. Static and dynamic postural stability in veterans with combat-related mild traumatic brain injury. Gait Posture. 2015;42(42):550-557. doi:10.1016/j. gaitpost.2015.08.012

12. Gupta A, Ledin T, Larsen LE, Lennmarken C, Odkvist LM. Computerized dynamic posturography: a new method for the evaluation of postural stability following anaesthesia. $\mathrm{Br} J$ Anaesth. 1991;66:667-672.

13. Weiss A, Herman T, Giladi N, Hausdorff JM. Objective assessment of fall risk in Parkinson's disease using a body-fixed sensor worn for 3 days. PLoS One. 2014;9:e96675. doi:10.1371/journal.pone.0096675

14. Horak F, King L, Mancini M. Role of body-worn movement monitor technology for balance and gait rehabilitation. Phys Ther. 2015;95:461-470. doi:10.2522/ptj.20140253

15. Gago MF, Fernandes V, Ferreira J, et al. Postural stability analysis with inertial measurement units in Alzheimer's disease. Dement Geriatr Cogn Dis Extra. 2014;4:22-30. doi:10.1159/000357472

16. Craig JJ, Bruetsch AP, Lynch SG, Horak FB, Huisinga JM. Instrumented balance and walking assessments in persons with multiple sclerosis show strong test-retest reliability. $J$ Neuroeng Rehabil. 2017;14:43. doi:10.1186/s12984-017-0320-4

17. Angelaki DE, Cullen KE. Vestibular system: the many facets of a multimodal sense. Annu Rev Neurosci. 2008;31:125-150. doi:10.1146/annurev.neuro.31.060407.125555

18. Knoll RM, Ishai R, Trakimas DR, et al. Peripheral vestibular system histopathologic changes following head injury without temporal bone fracture. Otolaryngol Head Neck Surg. 2019;160(1):122-130.

19. Yates BJ. Autonomic reaction to vestibular damage. Otolaryngol Head Neck Surg. 1998;119:106-112. doi:10.1016/S0194-5998(98)70179-2
20. Fino PC. A preliminary study of longitudinal differences in local dynamic stability between recently concussed and healthy athletes during single and dual-task gait. J Biomech. 2016;49:1983-1988. doi:10.1016/j.jbiomech.2016.05.004

21. Grooms DR, Onate JA. Neuroscience application to noncontact anterior cruciate ligament injury prevention. Sports Health. 2016;8:149-152. doi:10.1177/1941738115619164

22. Lynall RC, Mauntel TC, Padua DA, Mihalik JP. Acute lower extremity injury rates increase after concussion in college athletes. Med Sci Sports Exerc. 2015;47:2487-2492. doi:10.1249/ MSS.0000000000000716

23. Swanik CB, Covassin T, Stearne DJ, Schatz P. The relationship between neurocognitive function and noncontact anterior cruciate ligament injuries. Am J Sports Med. 2007;35:943-948. doi:10.1177/ 0363546507299532

24. Wilkerson GB, Grooms DR, Acocello SN. Neuromechanical considerations for postconcussion musculoskeletal injury risk management. Curr Sports Med Rep. 2017;16:419-427. doi:10.1249/ JSR.0000000000000430

25. Beleckas C, Calfee R. Distal radius fractures in the athlete. Curr Rev Musculoskelet Med. 2017;10:62-71. doi:10.1007/s12178-0179385-8

26. King LA, Mancini M, Fino PC, et al. Sensor-based balance measures outperform modified balance error scoring system in identifying acute concussion. Ann Biomed Eng. 2017;45:2135-2145. doi:10.1007/s10439-017-1856-y

27. Maurer C, Peterka RJ. A new interpretation of spontaneous sway measures based on a simple model of human postural control. J Neurophysiol. 2005;93:189-200. doi:10.1152/jn.00221.2004

28. Yamamoto T, Smith CE, Suzuki Y, et al. Universal and individual characteristics of postural sway during quiet standing in healthy young adults. Physiol Rep. 2015;3:e12329. doi:10.14814/ phy2.12329

29. Nashner LM, Shupert CL, Horak FB, Black FO. Organization of posture controls: an analysis of sensory and mechanical constraints. Prog Brain Res. 1989;80:411-418.discussion 395-397.

30. Palmieri RM, Ingersoll CD, Stone MB, Krause BA. Center-ofpressure parameters used in the assessment of postural control. J Sport Rehab. 2002;11:51-66. doi:10.1123/jsr.11.1.51

31. Neville C, Ludlow C, Rieger B. Measuring postural stability with an inertial sensor: validity and sensitivity. Med Devices (Auckl). 2015;8:447-455. doi:10.2147/MDER.S91719

32. Seimetz C, Tan D, Katayama R, Lockhart T. A comparison between methods of measuring postrual stability: force plates versus accelerometers. Biomed Sci Instrum. 2012;48:386-392.

33. Whitney SL, Roche JL, Marchetti GF, et al. A comparison of accelerometry and center of pressure measures during computerized dynamic posturography: a measure of balance. Gait Posture. 2011;33:594-599. doi:10.1016/j.gaitpost.2011.01.015

34. Sankarpandi SK, Baldwin AJ, Ray J, Mazza C. Reliability of inertial sensors in the assessment of patients with vestibular disorders: a feasibility study. BMC Ear Nose Throat Disord. 2017;17:1. doi:10.1186/s12901-017-0034-z

35. Le Flao E, Hume P, King D. Head impact monitoring: what new methodologies could do for concussion biomechanics. ISBS Proc Arch. 2018;36(Article):257.

36. Salisbury JP, Keshav NU, Sossong AD, Sahin NT. Standing balance assessment using a head-mounted wearable device. bioRxiv. 2017. doi:10.1101/149831

37. Thomas KS, Magal M. How does physical activity impact postural stability? J Nov Physiother. 2014;4:206.

38. Romberg MH. A Manual of the Nervous Diseases of Man. London: New Sydenham Society; 1853.

39. Lanska DJ, Goetz CG. Romberg's sign: development, adoption, and adaptation in the 19th century. Neurology. 2000;55:12011206. 
40. Pereira CB, Strupp M, Holzleitner T, Brandt T. Smoking and balance: correlation of nicotine-induced nystagmus and postural body sway. Neuroreport. 2001;12:1223-1226.
41. Seigle B, Ramdani S, Bernard PL. Dynamical structure of center of pressure fluctuations in elderly people. Gait Posture. 2009;30:223-226. doi:10.1016/j.gaitpost.2009.05.005

\section{Publish your work in this journal}

Medical Devices: Evidence and Research is an international, peerreviewed, open access journal that focuses on the evidence, technology, research, and expert opinion supporting the use and application of medical devices in the diagnosis, monitoring, treatment and management of clinical conditions and physiological processes. The identification of novel devices and optimal use of existing devices which will lead to improved clinical outcomes and more effective patient management and safety is a key feature of the journal. The manuscript management system is completely online and includes a very quick and fair peer-review system. Visit http:// www.dovepress.com/testimonials.php to read real quotes from published authors.

Submit your manuscript here: https://www.dovepress.com/medical-devices-evidence-and-research-journal 